\title{
Analysis of the barriers and enablers to implementing lifestyle management practices for women with PCOS in Singapore
}

Henry Ko ${ }^{1,2,3^{*}}$, Helena Teede ${ }^{4}$ and Lisa Moran ${ }^{4,5}$

\begin{abstract}
Background: Polycystic ovary syndrome (PCOS) is a condition that affects women of reproductive age and manifests with adverse reproductive, metabolic and psychological consequences. Evidence-based PCOS guidelines recommend lifestyle management first line for infertility. In Singapore women with PCOS can attend the PCOS Clinic at the Kandang Kerbau Women and Children's Hospital for infertility treatment. However lifestyle integration into infertility management is currently limited and barriers and enablers to progress remain unclear.

Methods: All PCOS clinic staff undertook semi-structured interviews to investigate perceived barriers for staff and consumers for the integration of lifestyle into infertility management. This study utilised various tools including an $8 \mathrm{P}$ Ishikawa diagram model to identify and categorise barriers. A modified Hanlon method was then used to prioritise barriers within the Singaporean context considering organisational, cultural and financial constraints. Propriety, economics, acceptability, resources and legality (PEARL) criteria were also incorporated into this decision-making tool.

Results: In the 8P model, there were five factors contributing to the 'procedure (consultations and referral processes)' barrier, one 'policy (government and hospitals)' factor, five 'place' factors, two 'product (lifestyle management programme)'barriers, two 'people (programme capacity)' factors, four 'process (integration)'factors, three 'promotion' barriers and three 'price' factors. Of the prioritised barriers, two were identified across each of 'procedures,' 'place,' 'product' and 'people' and four related to 'processes.' There were no barriers identified that for 'policies,', promotion' and 'price' that can be addressed.

Conclusions: There is a clear need to integrate lifestyle into infertility management in PCOS, in line with current national and international evidence-based guidelines. The highest priority identified improvement opportunity was to develop a collaborative lifestyle management programme across hospital services. Reductions in variation of delivery and strengthening support within the lifestyle programme are other identified priorities. The strength of this study is that this is the first study to utilise a pragmatic quality improvement method for barriers identification and prioritisation in the area of lifestyle management for women with PCOS. This project identified factors that may provide easy improvements, but also identified some local factors that may be very difficult to change. The major limitation of this study is that it is only looking at the Singapore setting, so may have limited applicability to other countries. However, results from quality improvement projects are meant to be context specific.
\end{abstract}

Keywords: Polycystic ovary syndrome, Lifestyle management, Clinical practice guideline, Barriers, Prioritisation

\footnotetext{
*Correspondence: henry.ko@ctc.usyd.edu.au

${ }^{3}$ NHMRC Clinical Trials Centre, University of Sydney, Levels 4-6,

Medical Foundation Building, 92-94 Parramatta Rd, Camperdown,

NSW 2050, Australia

Full list of author information is available at the end of the article
} 


\section{Background}

Polycystic ovary syndrome (PCOS) is a condition affecting up to $18 \%$ of reproductive-aged women [1]. It manifests with adverse reproductive (hyperandrogenism, menstrual dysfunction, infertility and pregnancy complications) [1], metabolic (insulin resistance, dyslipidaemia, non-alcoholic fatty liver disease, endothelial dysfunction, early atherosclerosis and increased impaired glucose tolerance, type 2 diabetes mellitus and cardiovascular disease) [2], and psychological (worsened quality of life and increased anxiety and depression) [3]. Overweight and obesity are strongly related to PCOS with higher energy intake and rates of weight gain driven by hormonal and other factors $[4,5]$. Obesity then increases the prevalence and severity of reproductive, metabolic and psychological morbidity in PCOS. PCOS has therefore been identified by the Australian National Women's Health Policy as a key obesity-related reproductive problem [6].

The first national and international, evidence-based clinical practice guideline (CPG) for the management of PCOS was developed by the PCOS Australian Alliance and approved by the Australian National Health and Medical Research Council in 2011 [7]. The CPG was auspiced by the Jean Hailes Foundation for Women's Health and supported by the consumer advocacy group Polycystic Ovary Syndrome Association of Australia. A key clinical priority of the CPG was lifestyle (diet, exercise or behavioural) management to optimise weight management (defined as the prevention and treatment of excess weight) and the reproductive, metabolic and psychological features of PCOS delivered through multidisciplinary integrated care. Lifestyle management was also prioritised for the treatment of infertility in PCOS.

Obesity is common and is increasing in Singapore with $10.8 \%$ of adults obese $\left(\mathrm{BMI} \geq 30 \mathrm{~kg} / \mathrm{m}^{2}\right)(9.5 \%$ of females) and $23 \%$ in the high risk Asian BMI category $\left(B M I \geq 27.5 \mathrm{~kg} / \mathrm{m}^{2}\right)(21.4 \%$ of females) [8, 9]. While the incidence of PCOS in Singapore is unclear, the prevalence of Rotterdam defined PCOS internationally spans from 6 to $18 \%$ depending on the BMI, ethnicity and diagnostic criteria used $[10,11]$. Both obesity and PCOS contribute to reduced fertility as does advanced maternal age and changing work and lifestyle commitments amongst women. Possible lack of knowledge about these influencing factors including PCOS may be impacting on lower birth rates in Singapore.

The Singaporean government has a focus on increasing the birth rate and has made fertility a national issue. Infertility is commonly treated through assisted reproductive technologies (ART) [including in vitro fertilisation (IVF)]. The 2014 clinical practice guideline released by the Singapore Ministry of Health on management of infertility recommended weight reduction as first line treatment (i.e. before ART) as a good practice point [12], however more specific recommendations, methods and evidence were not elaborated upon. The Singapore Health Promotion Board is a government organisation that actively promotes healthy living and promotes lifestyle advice resulting in a very visible social presence regarding weight management in Singapore.

Given the current evidence in this field, the recommendations of international evidence-based guidelines and the Singapore guidelines and government focus on addressing increasing infertility highlighting the importance of weight reduction, there is increasing acknowledgement of the need to improve the integration of lifestyle management into PCOS clinical infertility management. Most ART clinics in Singapore are located in the major hospitals, with some located in private facilities. One of the major fertility and ART clinics in Singapore is at the Kandang Kerbau Women's and Children's Hospital's (KKH) IVF Clinic. The KKH PCOS Clinic is co-located within the KKH IVF Clinic and provides advice to women with PCOS seeking pregnancy. The clinic consists of medical and allied health staff, including reproductive endocrinologists, dietitians and exercise physiologists. The KKH PCOS Clinic has acknowledged the need to improve the delivery of lifestyle management programmes. Clinicians identified some local problems with the programme, including: (1) a lack of a coordinated approach to integration of lifestyle, (2) a possibly low adoption rate, (3) a low sustainability rate once started, (4) uncertainty around optimal organisation, resourcing and patient support and (5) a lack of a clear follow-up and monitoring process and documentation for those women dropping out. These factors limited the confidence of local providers to integrate a lifestyle focus into infertility management.

A proposal was made to test and translate the recommendations from the rigorously developed Australian PCOS lifestyle management CPG to the Singaporean setting. This project aimed to determine what the key barriers were to women with PCOS taking up and adhering to a lifestyle management programme and to determine what programme adaptations could improve sustainability. As far as the authors are aware this is the first time a formal barriers and enablers analysis for integration of lifestyle management into infertility management for women with PCOS has been published.

\section{Methods}

This project used methodology based on a quality improvement framework. The problems and barriers to optimal implementation of a lifestyle management programme were identified as a first step to change and implementation. 
The pragmatic QI accelerated model for improvement (AMI) methodology promoted by SingHealth department of Clinical Governance and Quality Management, Profound Knowledge Partners Inc [13], and Associates in Process Improvement $[14,15]$ was used for this exercise (Fig. 1). A key concept in the project selection and resourcing stage are four fundamental ways to implement change:

(1) redesign an existing product, process or service,

(2) design a new product, process, or service,

(3) improve the system as a whole and

(4) collaborate and share known solutions to test and modify into other parts of the system.

The activities for project objectives and measures was to document: (1) the project description, (2) the current situation, (3) boundaries, (4) the plan-do-study-act (PDSA) cycles to develop and test changes, (5) identify the goals to be achieved and (6) identify the measures to that can be used to prove success (or failure). PDSA are a QI method where multiple parallel cycles can be used to speed up testing, results, and learning.

The first step was to undertake process mapping or to map the current patient flows and decision-making touch-points for the patient journey within the $\mathrm{KKH}$ PCOS and IVF Clinic. Problems and issues were identified through formal and informal face-to-face group and one-on-one semi-structured meetings discussions with all KKH PCOS Clinic clinicians and allied health staff. This included three reproductive medicine and IVF specialists, one endocrinologist, two nutritionists, one nurse, and one exercise physiologist. These staff are representative of all disciplines involved in the KKH PCOS Clinic team with other health care workers. We used the National Institute of Clinical Studies (NICS) guide [16] to identify barriers to evidence uptake by staff during these discussions through techniques including brainstorming, using key informants and interviews and the NICS barrier tool [17]. We also utilised a barrier identification and

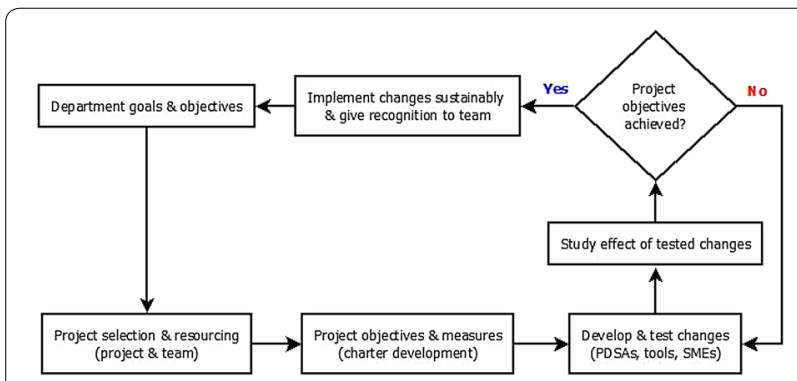

Fig. 1 AMI process summary mitigation (BIM) tool which is used when implementing CPGs as a secondary method for identifying barriers [18]. Due to hospital privacy restrictions, no patients were interviewed for this research.

Problems and issues specific to lifestyle management were structured into an Ishikawa diagram to identify key factors that could be barriers to women taking up and sustaining lifestyle management for their PCOS. An Ishikawa diagram, also known as a fishbone, herringbone, or cause-and-effect diagram, is used to map out the causes of a specific event. It is used in QI research to conceptually and visually group various causes of a problem together into major categories. We used the 8P model commonly used in the service industry as the major categories. The categories are: procedures, policies, place, product, people, processes, price and promotion. 'Procedures' related to consultation and referral processes, 'policies' related to government and hospital policies, 'place' related to Singapore-specific cultural factors, 'product' related to the lifestyle management programme, 'people' related to the programme capacity and staffing, 'processes' related to the integration and coordination of the programme internally and externally to other healthcare services, 'promotion' related to how lifestyle management was promoted to stakeholders and 'price' related to the financial aspects of PCOS treatments and lifestyle management. The Ishikawa diagram was the main tool to map the barriers and enablers to uptake and sustainability of lifestyle management for women with PCOS in Singapore.

Barriers identified in the Ishikawa diagram were then considered for a priority-setting exercise to determine which barriers should be tackled first with QI methods. We modified the Hanlon method [19] for prioritising health problems to prioritise barriers that we could address pragmatically within the Singaporean setting given the organisational, cultural and financial constraints with only barriers achieving a Hanlon method score $>13.5$ (the possible median score) considered for future improvement initiatives and QI PDSA development. The modified Hanlon Method rates things based on the sum of three scored criteria-size, seriousness, and effectiveness. This study used a simple 'yes', 'maybe', or 'no' decision to score to what extent each of the identified barriers satisfied each of the modified Hanlon method criteria. The higher the total score (i.e. the more 'yes'), the more priority the particular barrier has.

We modified the Hanlon Method scoring and problem considerations for our project for the size, seriousness and effectiveness (SSE) criteria (i.e. originally the Hanlon method is for prioritising health problems) to consider the perspective of health service programme delivery and resourcing. The effectiveness criteria ranking for the 
intervention was ranked by considering the PEARL criteria score, which is further explained later. The Hanlon method for getting the total score for the SSE criteria used the following formula:

$$
\mathrm{D}=[\mathrm{A}+(2 \times \mathrm{B}) \times \mathrm{C}]
$$

where: $\mathrm{D}=$ priority score $\mathrm{A}=$ size of problem ranking, $\mathrm{B}=$ seriousness of problem ranking, $\mathrm{C}=$ effectiveness of intervention ranking.

Note: according to the Hanlon method, the seriousness of the problem is weighted twice as much as the size of the problem, hence the " $2 \times \mathrm{B}$ " [19].

For the KKH PCOS Clinic and PCOS in Singapore, the modified Hanlon method scoring considered the following factors:

- 'Size': refers to size of the problem for PCOS women.

- 'Seriousness': refers to the urgency, demand, economic impact, QoL impact, and adverse health outcomes of this issue for PCOS women.

- 'Effectiveness': refers to the predicted effectiveness of any initiatives on addressing the problem. (Note: Effectiveness is based on the PEARL scores).

The maximum possible score is 27 . The minimum possible score is zero. The median score is 13.5 . It is the modified Hanlon method scores that determine which barriers identified in the Ishikawa diagram are deemed the most important to tackle.

To score the "effectiveness" component of the modified Hanlon method, the authors used a proxy measure to gauge the hypothetical feasibility, importance and impact of tackling the identified barriers. This was done by using the commonly used "PEARL criteria". The PEARL criteria uses the domains of propriety, economics, acceptability, resources, and legality (PEARL) to identify and rank pragmatic 'operational' issues of any barriers and interventions we were proposing to test [19]. The details and explanations on scoring the PEARL criteria are reported in the Additional file 1: Tables S1. Briefly, the analysis rated how each identified barrier could be classified under each PEARL criteria. It is important to note that these criteria were considered primarily from a hospital management and resourcing perspective, and not necessarily from a national public health perspective. For each factor the following questions are considered:

- 'Propriety': Is a program for tackling this problem suitable?

- 'Economics': Does it make economic sense to address this problem? Are there economic consequences if this problem is not tackled?
- 'Acceptability': Will the medical and patient community accept the program? Is it wanted, and by whom?

- 'Resources': Is funding available or potentially available for a solution?

- 'Legality': Do current laws or corporate management allow proposed solutions to be implemented?

This study used a simple 'yes', 'maybe', or 'no' decision to score to what extent each of the identified barriers satisfied each of the PEARL criteria. Any items that scores at least one "No" will be least prioritised. The more 'yes' a barrier had, the higher the total score, and therefore the more important and feasible it may be to tackle that particular barrier with a QI approach. The maximum possible total is 18 . The minimum possible total is five. The median score is 11.5 .

This pragmatic prioritisation method mostly used information from discussions with the PCOS Clinic staff and understanding of the clinical setting and political/ organisational context to inform the rankings, with the scoring providing a semi-quantitative component.

\section{Results}

There are three patients groups referred to the $\mathrm{KKH}$ PCOS Clinic from the KKH Infertility or KKH IVF Clinics-patients who have infertility problems (Additional file 1: Figure S1), menstrual problems (Additional file 1: Figure S2), and/or ovulation problems (Additional file 1: Figure S3). Of these, women who are overweight can be recommended to a lifestyle management programme. The lifestyle management programme consists of a set of three diet and one psychology sessions consisting of goal setting and physical activity counselling. There is also follow-up with exercise physiologists, dietitians, and clinicians.

Discussions with key KKH PCOS Clinic staff revealed a generally low uptake of the lifestyle programme as well as low sustainability of the programme. Staff reported that women usually opted for drug treatments and then ART, either immediately or after prematurely dropping out of the lifestyle programme.

Using the NICS guide [16] and NICS barrier tool [17] all identified barriers and issues were structured into an Ishikawa diagram " $8 \mathrm{P}$ model" with the important barriers, determined from the ranking exercise, highlighted (Fig. 2). A more detailed Ishikawa diagram is available in the Supplementary materials online (Additional file 1: Figure S4).

Table 1 shows the key results for the PEARL and modified Hanlon Method ranking exercise, which indicates the which identified barriers may be considered for future QI PDSAs. Of these, two (out of four) barriers have to do 


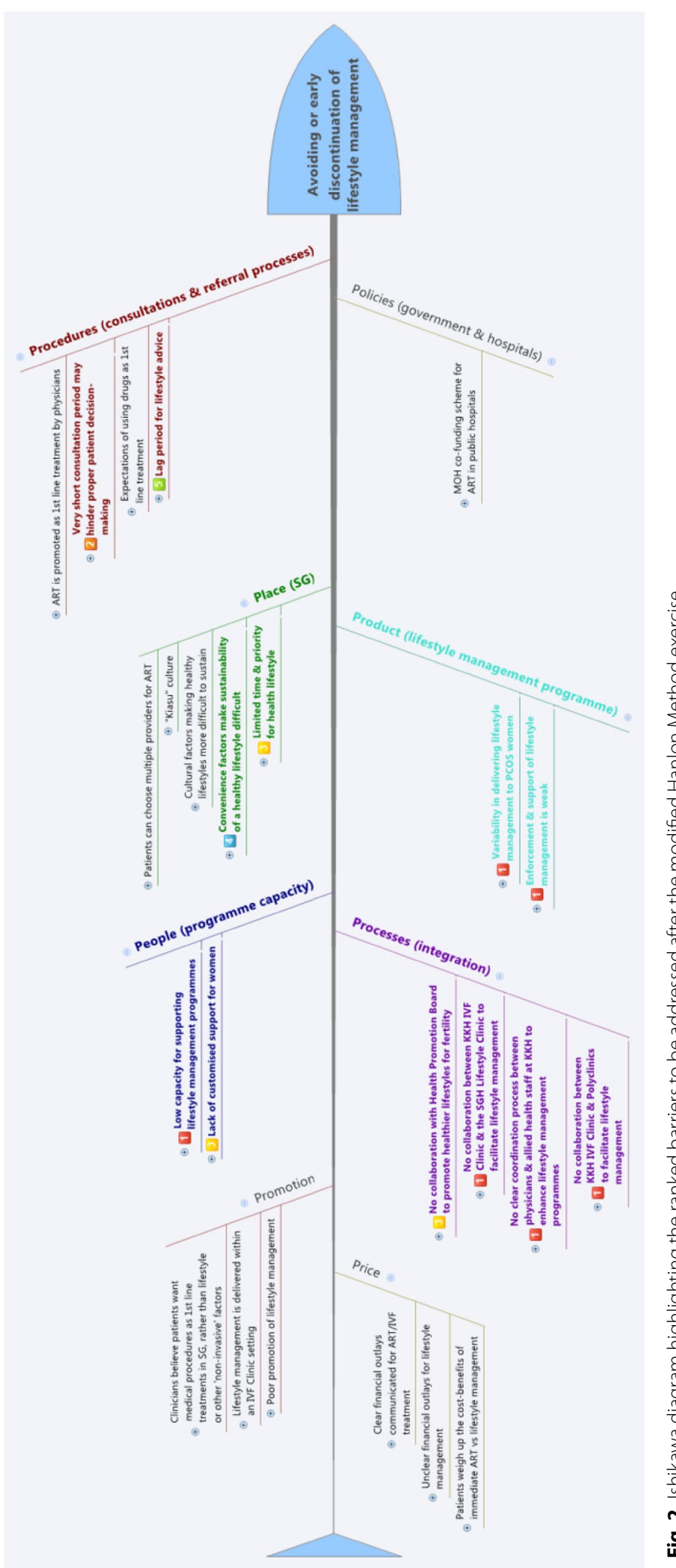

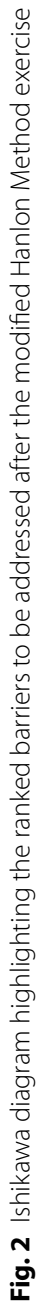




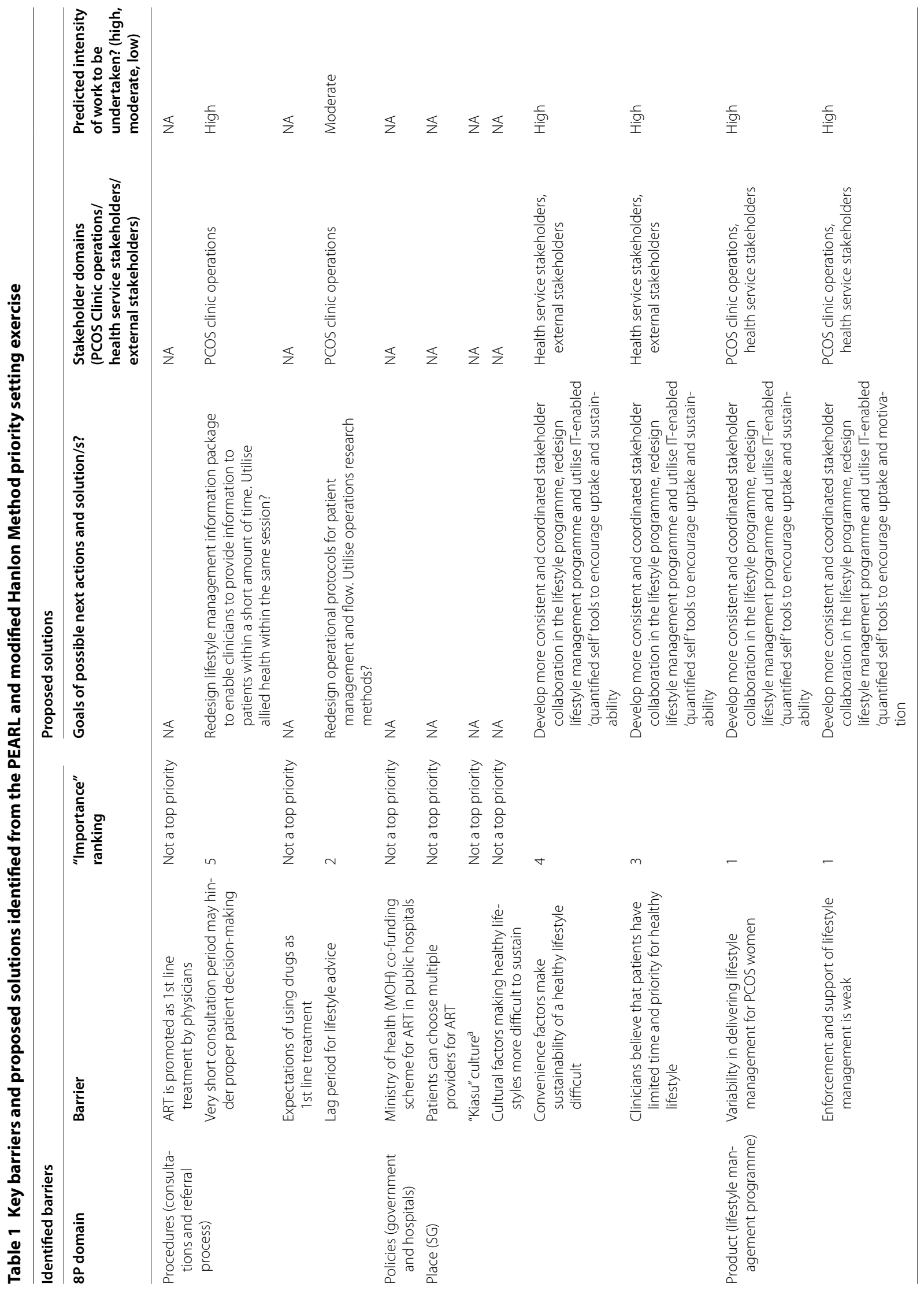



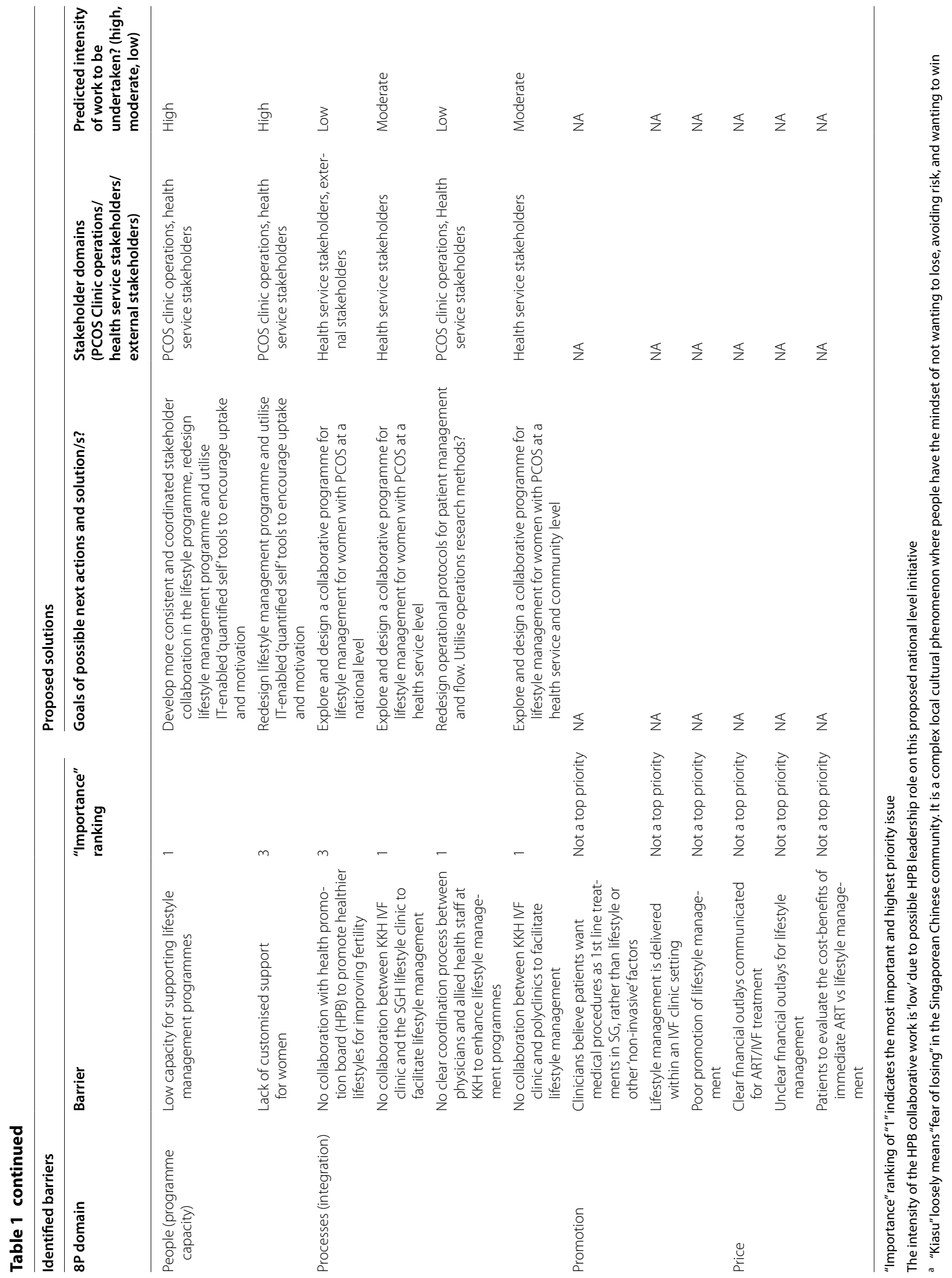
with 'procedures (consultations and referral processes)', two (out of five) relate to place, two (out of two) barriers relate to 'product (lifestyle management programme)', two (out of two) barriers relate to 'people (programme capacity)', and four (out of four) barriers relate to 'processes (integration)'. There were no barriers that were prioritised for 'policies (government and hospitals)', 'promotion' and 'price.' Table 1 also identifies the stakeholders that may be affected, the goals of these initial QI PDSAs, and the predicted intensity of the initiatives to be undertaken. More detailed explanations and scoring for each of the identified barriers and how these were ranked for Table 1, can be found in the Additional file 1: Tables S1.

It is noted that the highest priority (i.e. ranked \#1) was given to exploring collaborative lifestyle management programmes with the Singapore General Hospital (SGH) Lifestyle Clinic, Polyclinics (i.e. multidisciplinary primary care clinics, which may include allied health and dental care services), and between clinicians and allied health staff in $\mathrm{KKH}$ as a matter of improving the current lifestyle management programme "process (integration)". The other \#1 ranked priority was to manage the variability in delivering lifestyle management, and to strengthen and increase the support offered to women in the existing lifestyle programme.

\section{Discussion}

While recent evidence-based guidelines in PCOS highlight the key role of weight management through lifestyle programmes prior to pharmacological management and ART [20-22], there are clear gaps in current care. This study is the first to assess the barriers to implementation of an evidence-based clinical model integrating lifestyle management in infertility treatment in PCOS through identifying prioritising clinician-perceived barriers to integrating lifestyle programmes into infertility management for women with PCOS in Singapore. Barriers included factors relating to procedures (consultations and referral processes),' 'place,' 'product (lifestyle management programme)', 'people (programme capacity)', and 'processes (integration).' The barriers of 'policies (government and hospitals),' 'promotion' and 'price' were deemed by study participants as not feasible to tackle without extensive political effort.

Factors that may be relatively easy to tackle are interrelated factors of 'product', 'people' and 'process.' The highest priority in this analysis was given to the "process (integration)" barrier of improving the current lifestyle management programme through exploring collaborations with existing programmes and between clinicians and allied health staff as utilised in prior lifestyle management programs and recommended in the CPG [23-25]. From an organisational perspective, synergising and collaborating off existing initiatives would draw upon existing capabilities from each of these stakeholders and eliminate and reduce the duplication of existing programs. The other highly ranked priorities were to reduce the variability in delivering lifestyle management and increase the support offered in the lifestyle management programme. Enhancing multidisciplinary care co-ordination and integration between care providers along the patient journey are key concepts in health service delivery and prioritised by SingHealth and SGH's Department of Family Medicine and Continuing Care. This could be achieved in this setting through partnership with the SGH LIFE Centre which provides multidisciplinary and coordinated programmes for patients to manage lifestylerelated medical disorders and was Singapore's first hospital-based integrated and holistic lifestyle improvement facility. Multi-modal initiatives should also be designed to optimise collaborative programmes [26]. For example, existing healthier eating initiatives such as the Healthier Choice Symbol Programme for packaged foods and encouraging the provision of healthier meals at hawker centres and other food outlets is common in Singapore [27].

An identified 'place' barrier was the clinician perception that patients have limited time and priority for a healthy lifestyle. Strategies identified for optimising diet and exercise in other populations include the use of IT-enabled lifestyle management tools (i.e. e-health or $\mathrm{m}$-health tools) [28-34]. These may be particularly suitable in this population given the high education levels [35], high smartphone penetration and app usage [36, 37] and existing apps for accessing health information and health monitoring utilised by SingHealth (e.g. Health Buddy, Women and Child HealthPedia, Rheumatoid Arthritis app). These tools may also be preferable compared to face-to-face consultations in this specific population of reproductive-aged women with likely significant personal, work and family priorities [38]. However, testing and auditing of the actual use and effectiveness of these technologies on patients is required before large investments are made.

The barriers that were perceived as relatively difficult to tackle, although not impossible to change, were 'procedures' and 'place' which were both mainly related to the process of recommending treatment to women. For example, clinical staff thought that there was an expectation amongst Singaporean patients that drugs will be first-line treatment ('procedure' factor) and that patients were too busy and preferred to opt for treatment with a medication that may provide immediate results rather than spend months on lifestyle treatments ('place' factor). It is likely that the 'place' barriers are inherent to the local setting and may include convenience factors that could 
make sustainability of a healthy lifestyle difficult. Singapore has a high availability of fast food outlets [39] and a culture of eating low-cost street food at hawker centres and reduced home-based food preparation [40]. Opportunities for incidental activity may also be decreased in Singapore due to limited commuting distances related to the dense high-rise built environment and cheap transport. While these barriers cannot be easily addressed unless serious public health promotion efforts are made, a number of efforts are underway to tackle these problems in Singapore [26]. Ethical aspects of implementing lifestyle programme improvements were also highlighted, including a potential disincentive for progressing lifestyle integration into care related to financial incentives for ART clinicians to recommend ART instead of lifestyle management.

QI has been previously used for the assessment of managing obesity-related conditions. However, prior research has to date only described pre-post program implementation results [41, 42], with limited research reporting the specific QI methods used [43]. International databases for use in quality improvement studies include internationally recognised healthcare quality improvement institutions, such as the USA's Institute for Healthcare Improvement, UK NHS Institute for Innovation and Improvement (which closed in 2013) and Australia's NSW Agency for Clinical Innovation which contain databases on quality improvement case studies on healthcare initiatives including obesity management [44]. However, the focus has traditionally been for acute care problems rather than on the management of lifestyle and obesityrelated conditions with no assessment of PCOS. There is therefore a greater need to utilise and report quality improvement principles used in lifestyle management programs for PCOS because of various complex and interacting factors leading to typically poor attrition and sustainability [45].

The strengths of this study include that it: (1) is the first study to use a QI framework and tools for addressing barriers to implementation of programs in lifestyle management specifically in PCOS and (2) explicitly reports on the methods used to prioritise barriers to address for improvement specific for the Singaporean healthcare setting. One limitation of this study is that the process maps describing the PCOS patient journey may not take into consideration special cases that may occur. The maps were created with input from clinicians about the most common management pathways. The current PEARL and Hanlon prioritisation exercise was performed by "external observers" (i.e. QI/health services research staff) to try to avoid biases and conflicts of interest. Different groups may have differentially prioritised the barriers and enablers. Future research should therefore involve a comparison of these results to those from PCOS Clinic staff, patients, hospital administrators, government officials, or other stakeholders. The seniority and status of ART clinicians compared with allied health staff also needs to be addressed in future research to avoid professional conflicts in the prioritisation of staff opinions. Future research should also engage the key stakeholder groups of patients and health care consumers to identify barriers and assess consistency with clinician-identified barriers, particularly for the elements that patients experience (e.g. 'place', 'promotion', 'product'), as well as to help plan, test, and evaluate the strategies for lifestyle management.

The main implications of this study, identified from the QI methodologies, are that: (1) it is important to identify what barriers may or may not be reasonably be address (due to costs, stakeholder buy-in, resource limitations, systems, etc.), (2) financial and resource barriers need to be identified and prioritised from the beginning, (3) government policy and reimbursement "contradictions" may be barriers that are outside the scope of improvement projects. In our setting, government and reimbursement policies were factors that the research team felt were beyond the scope of future QI projects. For this reason, this issue has been ranked as a very low priority area. As the next phase of research, the authors are proposing a number of QI PDSAs to the KKH PCOS Clinic for testing to improve the lifestyle management service offered to patients. This will include addressing the 'process' (integration) barriers and to focus on coordinating and building a collaborative PCOS lifestyle management programme that works with other existing lifestyle facilities at SingHealth (e.g. SGH LIFE Centre). Once the collaboration is operational, patients and other stakeholders will be consulted to identify areas to further customise and improve the PCOS lifestyle management programme to optimise the 'Product' (i.e. the lifestyle management programme).

\section{Conclusions}

While CPG require implementation and evaluation strategies [46-48] and continuous quality improvement [49, 50] to drive evidence into practice, these activities are often limited and there is no prior research assessing these strategies for women with PCOS in general, and particularly for Singapore. Furthermore, while the recent Singaporean infertility clinical practice guidelines [12] contained recommendations on lifestyle management for PCOS, there was little consideration as to the optimal means of implementation within the current health care system. This research will therefore aid in the practical implementation of these recommendations. This study prioritised key barriers to lifestyle management to 
address including the exploration of collaborative lifestyle management programmes, reducing variability in delivering lifestyle management and increasing the support offered in the lifestyle management programme.

\section{Additional file}

Additional file 1. KKH PCOS Clinic patient management clinical algorithms.

\section{Authors' contributions}

HK conceived this project and carried out the design of this project, interviews, barriers analysis, and prioritisation and enablers analysis. LM participated in the design of this project, interviews, and prioritisation work. HT participated in the enablers analysis and drafting the manuscript. All authors read and approved the final manuscript.

\begin{abstract}
Author details
${ }^{1}$ SingHealth Centre for Health Services Research, Singapore Health Services Pte Ltd, 20 College Road, The Academia, Discovery Tower, Level 7 Translational and Clinical Research Hub, Singapore 169856, Singapore. ${ }^{2}$ SingHealth and Duke-NUS Academic Medicine Research Institute, Duke-NUS Graduate Medical School, Academia, Singapore Health Services Pte Ltd, 20 College Road, Singapore 169856, Singapore. ${ }^{3}$ NHMRC Clinical Trials Centre, University of Sydney, Levels 4-6, Medical Foundation Building, 92-94 Parramatta Rd, Camperdown, NSW 2050, Australia. ${ }^{4}$ Monash Centre for Health Research \& Implementation, School of Public Health, Monash University, Level 1, 43-51, Kanooka Grove, Clayton, VIC 3168, Australia. ${ }^{5}$ The Robinson Research Institute, University of Adelaide, Norwich Centre, Ground Floor, 55 King William Road, North Adelaide, SA 5006, Australia.
\end{abstract}

\section{Acknowledgements}

We thank Dr. Tan Heng Hao and Dr. Veronique Viardot-Foucault from KKH IVF Centre for their clinical input into the project. We thank Dr. Shanker Pasupathy and Moh Yoke Ping from the SGH LIFE Centre, and Dr. Tan Lee Boo from SGH dietetics department, for their advice regarding lifestyle programmes at SingHealth. Dr. Ko thanks his colleagues from the SingHealth Centre for Health Services Research and SingHealth Clinical Governance and Quality Management department for their advice regarding analysis and QI in this project. Preliminary work from this paper was presented at the Guidelines International Network Conference in Melbourne, 2014 and we would like thank the session participants for their valuable feedback.

\section{Competing interests}

HK acknowledges a financial competing interest in that he was employed by Sing Health, where this project was undertaken. HK acknowledges that the publication of this work and its recommendations may affect the financial interests (i.e. financial loss) of the institution and the staff who are the subjects of this project due to the underlying recommendation to redirect paying patients to another treatment pathway.

LM and HT declare that they have non-financial competing interests (i.e. academic) in that they were part of the Australian national group who developed the first national (and international) evidence-based clinical practice guidelines for managing women with PCOS.

\section{Data availability statement}

The detailed data used to derive the prioritisation scores will be freely available upon request. The online supplementary tables provide details of scoring and reasons of all domains analysed in this paper and presented in the Ishikawa diagram.

\section{Ethics statement}

As this project was considered a scoping or feasibility exercise as part of regular hospital healthcare quality improvement work, it did not require ethics approval. It involved no human subjects, human material, or human data.
Received: 21 October 2015 Accepted: 31 May 2016

Published online: 16 June 2016

\section{References}

1. Jean Hailes Foundation for Women's Health. Challenges in diagnostic assessment of PCOS. Melbourne: Jean Hailes Foundation for Women's Health; PCOS Australian Alliance; 2011.

2. Jean Hailes Foundation for Women's Health. Assessment of cardiometabolic risk in PCOS. Melbourne: Jean Hailes Foundation for Women's Health; PCOS Australian Alliance; 2011.

3. Jean Hailes Foundation for Women's Health. Assessment of emotional wellbeing in PCOS. Melbourne: Jean Hailes Foundation for Women's Health; PCOS Australian Alliance; 2011.

4. Teede HJ, Joham AE, Paul E, Moran LJ, Loxton D, Jolley D, et al. Longitudinal weight gain in women identified with polycystic ovary syndrome: results of an observational study in young women. Obesity. 2013;21(8):1526-32. doi:10.1002/oby.20213.

5. Moran LJ, Noakes M, Clifton PM, Wittert GA, Le Roux CW, Ghatei MA, et al. Postprandial ghrelin, cholecystokinin, peptide $Y Y$, and appetite before and after weight loss in overweight women with and without polycystic ovary syndrome. Am J Clin Nutr. 2007;86(6):1603-10.

6. Australian Government Department of Health and Ageing. National Women's Health Policy 2010. In: Australian Government Department of Health and Ageing, editor. Canberra: Australian Government Department of Health and Ageing; 2010.

7. Jean Hailes Foundation for Women's Health. Evidence-based guideline for the assessment and management of polycystic ovary syndrome. Melbourne: Jean Hailes Foundation for Women's Health; PCOS Australian Alliance; 2011.

8. Epidemiology and disease control division. National health survey 2010, Singapore. In: Ministry of Health S, editor. Singapore: Ministry of Health; 2011. p. 1-200.

9. Goh L, Pang J. Obesity in Singapore, prevention and control. Singap Fam Phys. 2012;38(1):8-13.

10. Yildiz BO, Bozdag G, Yapici Z, Esinler I, Yarali H. Prevalence, phenotype and cardiometabolic risk of polycystic ovary syndrome under different diagnostic criteria. Hum Reprod. 2012;27(10):3067-73. doi:10.1093/humrep/ des232.

11. Li R, Zhang Q, Yang D, Li S, Lu S, Wu X, et al. Prevalence of polycystic ovary syndrome in women in China: a large community-based study. Hum Reprod. 2013;28(9):2562-9. doi:10.1093/humrep/det262.

12. Academy of Medicine Singapore. Assessment and management of infertility at primary healthcare level. Singapore: Ministry of Health Singapore; 2013.

13. Profound Knowledge Partners Inc. Profound Knowledge Partners Inc. Profound Knowledge Partners Inc. 2013. http://pkpinc.com/index.html. Accessed 9 Jan 2015.

14. Associates in Process Improvement. Associates in Process Improvement. Associates in Process Improvement. 2015. http://www.apiweb.org/. Accessed 9 Jan 2015.

15. Langley G, Moen R, Nolan K, Nolan T, Norman C, Provost L. The improvement guide: a practical approach to enhancing organizational performance. 2nd ed. San Francisco: Jossey-Bass; 2009.

16. Australian National Institute of Clinical Studies. Identifying barriers to evidence uptake. Melbourne: Australian National Institute of Clinical Studies; 2006.

17. Australian National Institute of Clinical Studies. The NICS barrier tool. Melbourne: Australian National Institute of Clinical Studies; 2006.

18. Gurses A, Murphy D, Martinez E, Berenholtz S, Pronovost P. A practical tool to identify and eliminate barriers to compliance with evidence-based guidelines. Jt Comm J Qual Patient Saf. 2009;35(10):526-32.

19. National Association of County and City Health Officials. Prioritization techniques for selecting Ql efforts: Guide to prioritization techniques. USA: National Association of County and City Health Officials; 2011.

20. Teede HJ, Misso ML, Deeks AA, Moran LJ, Stuckey BG, Wong JL, et al. Assessment and management of polycystic ovary syndrome: summary of an evidence-based guideline. Med J Aust. 2011;195(6):S65-112. 
21. Jean Hailes Foundation for Women's Health. Non-pharmacological firstline management of infertility in PCOS. Melbourne: Jean Hailes Foundation for Women's Health; PCOS Australian Alliance; 2011.

22. Jean Hailes Foundation for Women's Health. Lifestyle management in PCOS. Melbourne: Jean Hailes Foundation for Women's Health; PCOS Australian Alliance; 2011.

23. Helmink J, Kremers S, Van Boekel L, Van Brussel-Visser F, Preller L, De Vries $N$. The BeweegKuur programme: a qualitative study of promoting and impeding factors for successful implementation of a primary health care lifestyle intervention for overweight and obese people. Fam Pract. 2012;29(Suppl 1):i68-74. doi:10.1093/fampra/cmr056

24. De Feo P, Fatone C, Burani P, Piana N, Pazzagli C, Battistini D, et al. An innovative model for changing the lifestyles of persons with obesity and/ or Type 2 diabetes mellitus. J Endocrinol Investig. 2011;34(10):e349-54. doi:10.3275/7857.

25. Aguer C, Gavarry O, Gole Y, Boussuges A, Doyard P, Falgairette G. A 5-month weight-reduction programme has a positive effect on body composition, aerobic fitness, and habitual physical activity of severely obese girls: a pilot evaluation study. J Sports Sci. 2010;28(3):281-9. doi:10.1080/02640410903460734.

26. Foo LL, Vijaya K, Sloan RA, Ling A. Obesity prevention and management: Singapore's experience. Obes Rev. 2013;14(Suppl 2):106-13. doi:10.1111/ obr.12092.

27. Lee BL. Dietary guidelines in singapore. Asia Pac J Clin Nutr. 2011;20(3):472-6.

28. Khaylis A, Yiaslas T, Bergstrom J, Gore-Felton C. A review of efficacious technology-based weight-loss interventions: five key components. Telemed J E Health. 2010;16(9):931-8.

29. Lyon J. The effectiveness of online and mobile technologies for changing health behaviours. Evidence Snapshot. New Zealand: Agencies for Nutrition Action. 2013. http://www.ana.org.nz/sites/default/files/Final\%20 snapshot\%20on\%20mobile\%20technologies.pdf.

30. Nguyen B, Kornman K, Baur L. A review of electronic interventions for prevention and treatment of overweight and obesity in young people. Obes Rev. 2011;12(5):e298-314.

31. Stephens J, Allen J. Mobile phone interventions to increase physical activity and reduce weight. J Cardiovasc Nurs. 2013;28(4):320-9.

32. Vodopivec-Jamsek V, de Jongh T, Gurol-Urganci I, Atun R, Car J. Mobile phone messaging for preventive health care. Cochrane Database of Syst Rev. 2012. doi:10.1002/14651858.CD007457.pub2.

33. Free C, Phillips G, Galli L, Watson L, Felix L. The effectiveness of mobilehealth technology-based health behaviour change or disease management interventions for health care consumers: a systematic review. PLoS Med. 2013;10(1):e1001362.

34. Harris J, Felix L, Miners A, Murray E, Michie S, Ferguson E, et al. Adaptive e-learning to improve dietary behaviour: a systematic review and costeffectiveness analysis. Health Technol Assess. 2011;15(37):1-180.

35. TIMSS and PIRLS International Study Center. TIMSS 2011 Assessment. TIMSS International Database 2013.

36. Anjum Z. South Korea and Hong Kong beat Singapore in smartphone penetration: Ericsson. In: ClO Asia-Industries. Executive Networks Media Research, Singapore. 2013. http://www.cio-asia.com/tech/industries/ south-korea-and-hong-kong-beat-singapore-in-smartphone-penetration-ericsson/?page=1. Accessed 5 May 2015.

37. Networks Asia staff. Singapore smartphone and tablet penetration on the rise; app usage increasing. In: Networks Asia_application development. Questex Asia, Hong Kong. 2013. http://www.networksasia.net/article/ singapore-smartphone-and-tablet-penetration-rise-app-usage-increasing-1371518626. Accessed 5 May 2015.
38. Gokee-Larose J, Gorin AA, Wing RR. Behavioral self-regulation for weight loss in young adults: a randomized controlled trial. Int J Behav Nutr Phys Act. 2009:6:10. doi:10.1186/1479-5868-6-10.

39. Whitton C, Ma Y, Bastian AC, Fen Chan M, Chew L. Fast-food consumers in Singapore: demographic profile, diet quality and weight status. Public Health Nutr. 2014;17(8):1805-13. doi:10.1017/S1368980013001997.

40. Wang MC, Naidoo N, Ferzacca S, Reddy G, Van Dam RM. The role of women in food provision and food choice decision-making in Singapore: a case study. Ecol Food Nutr. 2014;53(6):658-77. doi:10.1080/03670244.20 14.911178

41. Chakkalakal R, Camp A, Magenheimer E, Savoye M, Lubsen J, Lucas G, et al. Preventing diabetes among Fair Haven families: a community-based approach to quality improvement. J Health Care Poor Underserved. 2012;23(3 Supplement):247-54. doi:10.1353/hpu.2012.0136.

42. Cygan H, Baldwin K, Chehab L, Rodriguez N, Zenk S. Six to success: improving primary care management of pediatric overweight and obesity. J Pediatr Health Care. 2014;28(5):429-37. doi:10.1016/j. pedhc.2014.02.002.

43. Brandt K, Booker J, McGrath J. Clinical quality improvement for identification and management of overweight in pediatric primary care practices. Clin Pediatr. 2013;52(7):620-7. doi:10.1177/0009922813480844.

44. Galicia T, Centre UHH. Reducing obesity with improved self-management support. In: Improvement Stories. Institute for Healthcare Improvement, USA. 2015. http://www.ihi.org/resources/Pages/ImprovementStories/ ReducingObesitywithlmprovedSelfMgmtSupport.aspx. Accessed $24 \mathrm{Apr}$ 2015.

45. Moran L, Hutchison S, Norman R, Teede H. Lifestyle changes in women with polycystic ovary syndrome. Cochrane Database of Syst Rev. 2011(7):CD007506. doi:10.1002/14651858.CD007506.pub3.

46. Drabsch T. Rural collaborative guideline implementation: Evaluation of a hub and spoke multidisciplinary team model of care for orthogeriatric inpatients - a before and after study of adherence to clinical practice guidelines. Aust J Rural Health. 2015;23(2):80-6. doi:10.1111/ajr.12139.

47. McLeod RS, Aarts MA, Chung F, Eskicioglu C, Forbes SS, Conn LG, et al. Development of an enhanced recovery after surgery guideline and implementation strategy based on the knowledge-to-action cycle. Ann Surg. 2015. doi:10.1097/SLA.0000000000001067.

48. Dexheimer JW, Borycki EM, Chiu KW, Johnson KB, Aronsky D. A systematic review of the implementation and impact of asthma protocols. BMC Med Inform Decis Mak. 2014;14:82. doi:10.1186/1472-6947-14-82.

49. Diamond LH. Local implementation of clinical practice guidelines and continuous quality improvement: challenges and opportunities. Semin Dial. 2000;13(6):364-8.

50. Gibbons RJ, Smith SC Jr, Antman E. American college of C, American heart A. American college of Cardiology/American heart association clinical practice guidelines: Part II: evolutionary changes in a continuous quality improvement project. Circulation. 2003;107(24):3101-7. doi:10.1161/01.CIR.0000079017.53579.9C.

\section{Submit your next manuscript to BioMed Central and we will help you at every step:}

- We accept pre-submission inquiries

- Our selector tool helps you to find the most relevant journal

- We provide round the clock customer support

- Convenient online submission

- Thorough peer review

- Inclusion in PubMed and all major indexing services

- Maximum visibility for your research

Submit your manuscript at www.biomedcentral.com/submit 